International Journal of Pure and Applied Mathematics

Volume $96 \quad$ No. 2 2014, 235-247

ISSN: 1311-8080 (printed version); ISSN: 1314-3395 (on-line version)

url: http://www.ijpam.eu

doi: http://dx.doi.org/10.12732/ijpam.v96i2.7

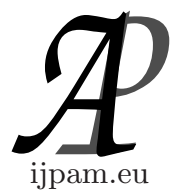

\title{
EXACT SOLUTIONS OF
}

\section{NAVIER STOKES EQUATIONS IN POROUS MEDIA}

\author{
Waqar Khan ${ }^{1}$, Faisal Yousafzai ${ }^{1}$, M. Ikhlaq Chohan ${ }^{2}$, \\ Anwar Zeb ${ }^{3}$, Gul Zaman ${ }^{4}$, Il Hyo Jung ${ }^{5}$ \\ ${ }^{1}$ School of Mathematical Sciences \\ University of Science and Technology of China \\ Hefei, 230026, P.R. CHINA \\ ${ }^{2}$ Department of Mathematics \\ Business Administration and Accounting \\ Buraimi University College \\ Al-Buraimi, OMAN \\ ${ }^{3}$ Department of Mathematics \\ COMSATS Institute of Information, Technology, Abbottabad, PAKISTAN \\ ${ }^{4}$ Department of Mathematics \\ University of Malakand \\ Chakdara Dir (Lower), Khyber Pakhtunkhwa, PAKISTAN \\ ${ }^{5}$ Department of Mathematics \\ Pusan National University \\ Geumjeong-Gu, Busan, 609-735, SOUTH KOREA
}

\begin{abstract}
This paper investigates exact solutions of steady Navier Stokes equations of an incompressible viscous fluid in a porous medium. Different flow situations are investigated using vorticity as a stream function. Making use of stream function, the two-dimensional flow equations are transformed into non-linear compatibility equation, and then it is linearized by vorticity function. Stream functions and velocity distributions are discussed for various flow situations.
\end{abstract}

Received: April 18, 2014

(C) 2014 Academic Publications, Ltd.

$\S$ Correspondence author url: www.acadpubl.eu 
AMS Subject Classification: 76S05, 76S99, 74A99

Key Words: porous medium, exact solutions, two-dimensional flows

\section{Introduction}

The basic governing equations for the flow of a fluid of Newtonian type are the Navier-Stokes equations. This set of non-linear partial differential equations has no general solution, and only a limited number of exact solutions can be found in literature. On the other hand, exact solutions are very important not only they represent the basic flow phenomena but also solutions obtained by various techniques can be attested by these basic solutions.

The study of the flow through porous media is indeed challenging because the flow phenomena and its possible applications in many areas such as: agricultural and irrigation sciences, oil and gas exploration, water resources, and many biological sciences etc. Flow models representing the flow through the porous media in this context are interesting and are of great importance. Our discussion on porous media flow cannot cover all of the different areas of application of flow through porous media. We are interested in investigating a simple flow model of the porous media known as the Darcy-Lapwood-Brinkman model. The Darcy-Lapwood-Brinkman equation in comparison with NavierStokes equations posses an extra term $\frac{\mu \mathbf{V}}{k}$, which shows resistance to the flow exerted by the porosity of the medium through which the fluid passes. This term has no effect on the non-linearity of governing partial differential equations. A number of papers in this direction are available $([1],[3],[4],[7])$. Hamdan [4], following Chandna [1], obtained solutions for the stream function and velocity vector in porous media, where he linearized the inertial term by a suitable choice of vorticity function.

In this paper, we investigate exact solutions of the Darcy Lapwood-Brinkman model for different flow situations. Solutions are found by using appropriate conditions for the different cases of flow. Expressions for the stream functions and velocity distributions are derived for two-dimensional flow behind a grid; flow above a porous plate; flow due to stretching plate, the corner flow and the asymptotic suction profile.

\section{Formulation of the Problem}

The steady flow of an incompressible viscous fluid through a porous medium is governed by the continuity and momentum equations by taking the Darcy- 
Lapwood-Brinkman model [4], given as

$$
\begin{gathered}
\nabla \cdot \mathbf{V}=\mathbf{0} \\
\rho(\mathbf{V} \cdot \nabla) \mathbf{V}=-\nabla p+\mu \nabla^{2} \mathbf{V}-\frac{\mu}{k} \mathbf{V}+\rho \mathbf{f},
\end{gathered}
$$

where $\nabla$ is the divergence, $\mathbf{V}$ the velocity vector, $\rho$ the constant density, $\mu$ the dynamic viscosity, $p$ the pressure, $k$ is the permeability, $\mathbf{f}$ the force vector and $\nabla^{2}$ denotes the Laplacian operator. We study the two-dimensional flow for which the velocity field is given by

$$
\mathbf{V}=[u(x, y), v(x, y), 0],
$$

where $u(x, y)$ and $v(x, y)$ are the velocity components along and normal to horizontal directions, respectively. Making use of equation (3) in equations (1) and (2) and neglecting the external forces, we find

$$
\begin{aligned}
u_{x}+v_{y} & =0, \\
P_{x}-\rho v \omega & =\mu \nabla^{2} u-\frac{\mu}{k} u, \\
P_{y}+\rho u \omega & =\mu \nabla^{2} v-\frac{\mu}{k} v,
\end{aligned}
$$

where

$$
\omega=v_{x}-u_{y}
$$

is the vorticity, and

$$
P=p+\frac{1}{2} \rho\left(u^{2}+v^{2}\right),
$$

is the generalized pressure. Equations (4-6) represent the continuity, and components of the momentum equation, respectively. Once the velocity components are determined, the pressure field in (8) is easily obtained by integrating (5) and (6). Introducing stream function $\psi(x, y)$ such that,

$$
u=\psi_{y}, v=-\psi_{x} .
$$

Therefore vorticity becomes

$$
\omega=-\nabla^{2} \psi
$$

Introducing (9) and (10) in equations (5), (6), (8) and eliminating pressure from equations (5) and (6) and by applying the integrability condition $P_{x y}=P_{y x}$, we get

$$
\rho\left[\psi, \nabla^{2} \psi\right]+\mu \nabla^{4} \psi-\frac{\mu}{k} \nabla^{2} \psi=0,
$$


and

$$
P=p+\frac{\rho}{2}\left(\psi_{y}^{2}+\psi_{x}^{2}\right)
$$

Here $\left[\psi, \nabla^{2} \psi\right]$, is the usual Poisson bracket and $\nabla^{4}=\nabla^{2} \cdot \nabla^{2}$.

Equation (11) is a non-linear partial differential equation of an incompressible viscous fluid in a porous medium. In order to linearize (11), vorticity is assumed of the form

$$
\omega=-\nabla^{2} \psi=\psi+A_{1} x+A_{2} y
$$

where $A_{1}$ and $A_{2}$ are constants. Substitution of (13) into (11) yields

$$
\nabla^{2} \psi+\frac{1}{v \beta}\left(A_{2} \psi_{x}-A_{1} \psi_{y}\right)=0
$$

where $v=\frac{\mu}{\rho}$ and $\beta=1-\frac{1}{k}$. Introduce

$$
\Sigma=\psi+A_{1} x+A_{2} y
$$

then equation (14) can be written as

$$
\nabla^{2} \Sigma+\frac{1}{v \beta}\left(A_{2} \Sigma_{x}-A_{1} \Sigma_{y}\right)=0
$$

which is a linear partial differential equation in $\Sigma$ and produces in a number of known exact solutions for different values of $A_{1}$ and $A_{2}$. Using the method of separation of variables and by setting

$$
\Sigma=f_{1}(x) f_{2}(y)
$$

Equation (16) becomes

$$
\begin{aligned}
& f_{1}^{\prime \prime}(x)+\frac{A_{2}}{v \beta} f_{1}^{\prime}(x)=C f_{1}(x), \\
& f_{2}^{\prime \prime}(y)-\frac{A_{1}}{v \beta} f_{2}^{\prime}(y)=-C f_{2}(y)
\end{aligned}
$$

in which $C$ is the separation constant, $f_{1}(x)$ and $f_{2}(y)$ are unknown functions to be determined. We now investigate the significance of this couple of linear equations in some special cases of fluid flow. 


\section{Exact Solutions for different Flow Geometries}

In this part of the paper, we investigate some exact solutions of the incompressible viscous fluid with porous medium for some physical flow situations considering proper values for constants $A_{1}, A_{2}$ and $C$. We determine stream functions and velocity fields for every case.

\subsection{Laminar Flow behind Two Dimensional Grid}

We consider the flow of an incompressible viscous fluid behind a two-dimensional grid with grid spacing $l$. For a desired solution of laminar flow behind a grid, we assume $A_{1}=0, A_{2}=-V$, and $C=\sigma^{2}>0$ where $V$ is the uniform velocity and $\sigma$ is a constant. Introducing these values of $A_{1}, A_{2}$ and $C$ in equations (18) and (19), we get

$$
\begin{aligned}
f_{1}^{\prime \prime}(x)-\frac{V}{v \beta} f_{1}^{\prime}(x)-\sigma^{2} f_{1}(x) & =0, \\
f_{2}^{\prime \prime}(y)+\sigma^{2} f_{2}(y) & =0,
\end{aligned}
$$

whose solutions are given as

$$
\begin{aligned}
& f_{1}(x)=B_{1} e^{m_{1} x}+B_{2} e^{m_{2} x} \\
& f_{2}(y)=B_{3} \cos (\sigma y)+B_{4} \sin (\sigma y)
\end{aligned}
$$

in which $B_{i}(i=1-4)$ are integration constants and

$$
m_{1,2}=\frac{1}{2}\left\{\frac{V}{v \beta} \pm \sqrt{\left(\frac{V}{v \beta}\right)^{2}+4 \sigma^{2}}\right\} .
$$

Substituting from equations (22) and (23) in equation (17), and assuming bounded solutions, we get

$$
\Sigma=M \sin (\sigma y) e^{\left\{\frac{V}{\sigma v \beta}-\sqrt{\left(\frac{V}{\sigma v \beta}\right)^{2}+4}\right\} \frac{\sigma x}{2}},
$$

in which the arbitrary constant $M=A_{2} A_{4}$ is to be determined. Making use of (15) and introducing non-dimensional parameters

$$
x^{*}=\frac{x}{l}, y^{*}=\frac{y}{l}, u^{*}=\frac{u}{V}, v^{*}=\frac{v}{V},
$$




$$
\psi^{*}=\frac{\psi}{V l}, M^{*}=\frac{M}{V l}, \sigma=\frac{2 \pi}{l},
$$

in $(25)$, we get

$$
\psi^{*}=y^{*}+M^{*} \sin \left(2 \pi y^{*}\right) e^{\gamma x^{*}},
$$

where $\gamma=\frac{\frac{R}{\beta}-\sqrt{\left(\frac{R}{\beta}\right)^{2}+16 \pi^{2}}}{2}$ and $R=\frac{V l}{\nu}$ is the Reynolds number. On setting $\beta \rightarrow 1$ we obtain the solution behind a two dimensional grid as shown by Kovasznay for the viscous fluid [5]. With the help of equation (9) the velocity components $u^{*}$ and $v^{*}$ are given as

$$
\begin{aligned}
& u^{*}=1+2 \pi M^{*} \cos \left(2 \pi y^{*}\right) e^{\gamma x^{*}}, \\
& v^{*}=\gamma M^{*} \sin \left(2 \pi y^{*}\right) e^{\gamma x^{*}} .
\end{aligned}
$$

The constant $M^{*}$ can be determined by setting the stagnation point of the flow where $\left(u^{*}, v^{*}\right)=(0,0)$. Suppose it is at $\left(x^{*}, y^{*}\right)=(0,0)$ then simplification yields $M^{*}=\frac{-1}{2 \pi}$ and

$$
\begin{aligned}
& u^{*}=1+\cos \left(2 \pi y^{*}\right) e^{\gamma x^{*}}, \\
& v^{*}=\frac{-\gamma}{2 \pi} \sin \left(2 \pi y^{*}\right) e^{\gamma x^{*}} .
\end{aligned}
$$

\subsection{Reverse Flow above a Plate}

Consider steady flow of an incompressible viscous fluid above a plate with suction at $y=0$. In order to study the behavior of the fluid above the plate it is assumed that $A_{1}=0, A_{2}=-V$, and $C=-\sigma^{2}<0$ where $V$ is the uniform velocity and $\sigma$ is a constant. Employing these values in equations (18) and (19), we obtain

$$
\begin{aligned}
f_{1}^{\prime \prime}(x)-\frac{V}{v \beta} f_{1}^{\prime}(x)+\sigma^{2} f_{1}(x) & =0, \\
f_{2}^{\prime \prime}(y)-\sigma^{2} f_{2}(y) & =0,
\end{aligned}
$$

Both these equations yield solutions of the form

$$
\begin{aligned}
& f_{1}(x)=E_{1} e^{n_{1} x}+E_{2} e^{n_{2} x}, \\
& f_{2}(y)=E_{3} e^{\sigma y}+E_{4} e^{-\sigma y},
\end{aligned}
$$

where $E_{i}(i=1-4)$ are arbitrary constants and

$$
n_{1,2}=\frac{1}{2}\left\{\frac{V}{v \beta} \pm \sqrt{\left(\frac{V}{v \beta}\right)^{2}-4 \sigma^{2}}\right\} .
$$


Therefore, with the help of equation (17) a bounded solution for $\Sigma$ becomes

$$
\Sigma=E \cdot e^{-\sigma y+\left\{\frac{V}{v \beta}+\sqrt{\left(\frac{V}{v \beta}\right)^{2}-4 \sigma^{2}}\right\} \frac{x}{2}}
$$

where $E=E_{2} E_{4}$ is a constant to be determined and $\sigma=\frac{2 \pi}{l}$, where $l$ is the length of the plate. The relation in equation (15) with the help of (26) yields a non-dimensional stream function of the form

$$
\psi^{*}=y^{*}+E e^{-2 \pi y^{*}+\vartheta x^{*}},
$$

where $\vartheta=\frac{\frac{R}{\beta}+\sqrt{\left(\frac{R}{\beta}\right)^{2}-16 \pi^{2}}}{2}$ and $E^{*}=\frac{E}{V l}>0$, represents suction at the plate. The velocity components are given by

$$
\begin{aligned}
& u^{*}=1-2 \pi E^{*} e^{-\left(2 \pi y^{*}+\vartheta x^{*}\right)}, \\
& v^{*}=\vartheta E^{*} e^{-\left(2 \pi y^{*}+\vartheta x^{*}\right)} .
\end{aligned}
$$

For $\beta \rightarrow 1$ these solutions are compatible with the solutions given by Lin and Tobak [6].

\subsection{Flow due to Stretching Plate}

We consider the two-dimensional boundary layer flow of a viscous fluid with porous medium above a stretching surface placed at $y=0$. The application of an external force along the $x$-direction results in stretching of the plate and hence the flow. The boundary conditions for the flow situation are:

$$
\begin{aligned}
& u(x, y)=\lambda x, u(0, y)=0, \\
& u(x, 0)=0, u(x, \infty)=-V,
\end{aligned}
$$

where $\lambda$ is the stretching parameter. For flow due to stretching of the plate, we assume $A_{1}=-V$ and $A_{2}=0=C$ in (15) and (16), then

$$
\begin{aligned}
f_{1}^{\prime \prime}(x) & =0, \\
f_{2}^{\prime \prime}(y)+\frac{V}{v \beta} f_{2}^{\prime}(y) & =0 .
\end{aligned}
$$

Solutions to both these equations are

$$
f_{1}(x)=E_{5} x+E_{6},
$$




$$
f_{2}(y)=E_{7}+E_{8} e^{\frac{-V}{v \beta} y},
$$

in which $E_{i}(i=5-8)$ are integration constants. Substituting from (45) and (46) and making use of (15) and (17) it is derived that

$$
\psi=V x+a_{1}+a_{2} x+a_{3} e^{\frac{-V}{v \beta} y}+a_{4} x e^{\frac{-V}{v \beta} y},
$$

where $a_{i}(i=1-4)$ are redefined constants of equations (45) and (46) to be determined. With the help of boundary conditions (41-43) and non-dimensional parameters (26), the stream function reduces to

$$
\psi^{*}=x^{*}-x^{*} e^{-\beta^{-1} R y^{*}},
$$

and the corresponding velocity components are as:

$$
\begin{aligned}
& u^{*}=\beta^{-1} R x^{*} e^{\left.-\beta^{-1} R y^{*}\right)}, \\
& v^{*}=e^{\left.-\beta^{-1} R y^{*}\right)}-1 .
\end{aligned}
$$

where $\lambda=\frac{V^{2}}{v \beta}$. If $\beta \rightarrow 1$ the velocity components reduce to the viscous case solutions given in Drazin and Riley [2].

\subsection{Flow into a Corner}

The flow of an incompressible viscous fluid along a corner is considered with suction at both the walls which are placed perpendicular to each other. The relevant boundary conditions are

$$
\begin{gathered}
u=v=0, \text { on } x=0, \text { and } y=0, \\
u, v \rightarrow-V, \text { as } x \rightarrow \infty, \text { and } y \rightarrow \infty .
\end{gathered}
$$

The constants $A_{1}, A_{2}$ and $C$ in (18) and (19) are given the values $-V, V$ and 0 respectively, where $V$ is the uniform velocity, then,

$$
\begin{aligned}
& f_{1}^{\prime \prime}(x)+\frac{V}{v \beta} f_{1}^{\prime}(x)=0, \\
& f_{2}^{\prime \prime}(y)+\frac{V}{v \beta} f_{2}^{\prime}(y)=0 .
\end{aligned}
$$

With the help of (15), (17) and solutions of the above equations lead to the following form of stream function

$$
\psi=V(x-y)+\left(E_{9}+E_{10} e^{\frac{-V}{v \beta} x}\right)\left(E_{11}+E_{12} e^{\frac{-V}{v \beta} y}\right),
$$


in which $E_{i}(i=9-12)$ are constants of integration. Using boundary conditions (51) and (52), we obtain expression for non-dimensional stream function as

$$
\psi^{*}=x^{*}-y^{*}+\beta R^{-1}\left[e^{-\beta^{-1} R x^{*}}-e^{-\beta^{-1} R y^{*}}\right],
$$

The velocity components become

$$
u^{*}=-\left(1-e^{-\beta^{-1} R y^{*}}\right), \quad v^{*}=-\left(1-e^{-\beta^{-1} R x^{*}}\right)
$$

we see that the no-slip conditions are satisfied at each boundaries, which are permeable and there is suction on both boundaries. For $\beta \rightarrow 1$ the above solutions recover the viscous flow solution given in [2].

\subsection{The Asymptotic Suction Profile}

Consider the flow of an incompressible viscous fluid in a porous medium over an infinite plate with uniform suction about $y=0$. Fluid with a uniform velocity is approaching towards the leading edge of the plate. The corresponding boundary conditions are

$$
\begin{aligned}
& u(x, y)=0 \text { at } y=0, \\
& u(x, y)=U \text { at } y \rightarrow \infty, \\
& u(x, y)=U \text { at } x=0, \\
& v(x, y)=-V \text { at } y=0, \\
& v(x, y)=0 \text { at } y \rightarrow \infty .
\end{aligned}
$$

The flow of this geometry can be described by assigning values $-V,-U$ and 0 to corresponding constants $A_{1}, A_{2}$ and $C$ respectively. Introducing these values into equations (18) and (19), we have

$$
\begin{aligned}
& f_{1}^{\prime \prime}(x)-\frac{U}{v \beta} f_{1}^{\prime}(x)=0, \\
& f_{2}^{\prime \prime}(y)+\frac{V}{v \beta} f_{2}^{\prime}(y)=0,
\end{aligned}
$$

Solving them and using (15) and (17), a stream function of the form is obtained

$$
\psi=V x+U y+\left(E_{13}+E_{14} e^{\frac{U}{v \beta} x}\right)\left(E_{15}+E_{16} e^{\frac{-V}{v \beta} x}\right),
$$



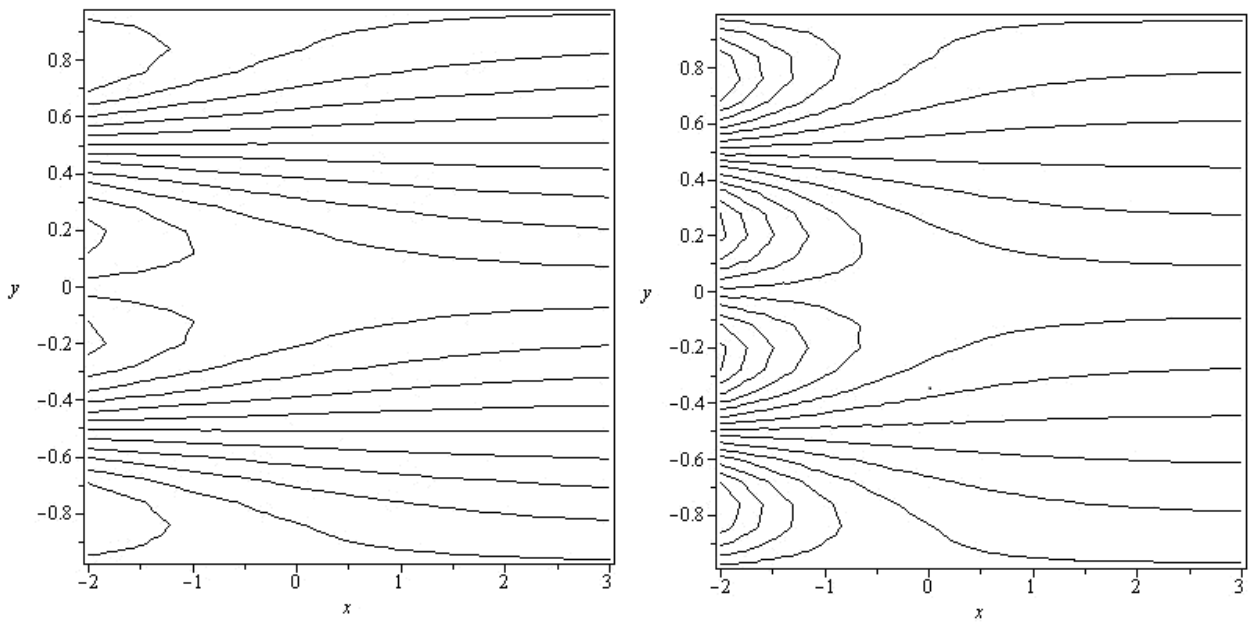

Figure 1: Stream lines for grid flow for $R=40$ and (a) $\beta=0.5$, (b) $\beta=1$

where $E_{i}(i=13-16)$ are constants of integration. Subject to boundary conditions (58-62) and using (26), the stream function becomes

$$
\psi^{*}=x^{*}+\varsigma y^{*}+\varsigma v \beta e^{\frac{R y^{*}}{\beta}},
$$

where $\varsigma=\frac{U}{V}$. The corresponding velocity components for (66) are

$$
\begin{aligned}
& u^{*}=\varsigma\left(1+e^{\frac{R y^{*}}{\beta}}\right), \\
& v^{*}=-1 .
\end{aligned}
$$

Similarly, in this case if $\beta \rightarrow 1$, then it represents the case of viscous fluid.

\section{Results and Discussions}

Expressions for the stream functions and velocity profile are dependent on the viscous fluid with permeability parameter Graphs have been plotted against choosing values of in such a manner that our results are verified by the viscous case solutions.

The stream lines pattern for the laminar flow behind a grid is shown in figure (1). It can be seen from the figure that bound eddies are developed 

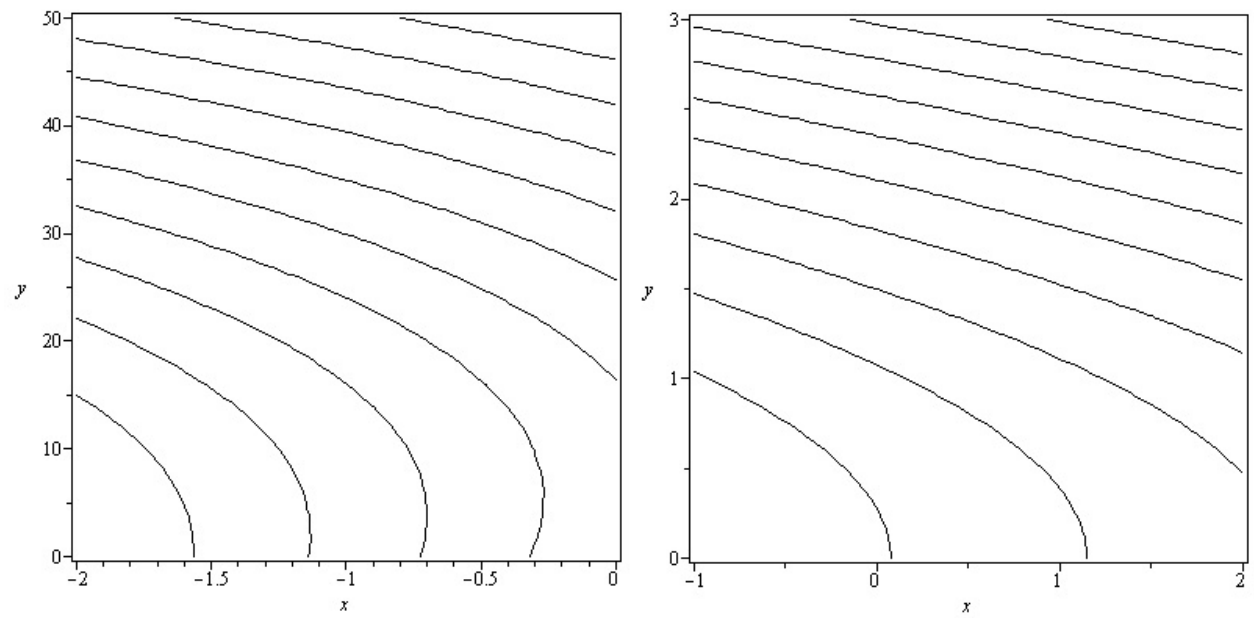

Figure 2: Stream lines for reverse flow pfor $R=13$ and (a) $\beta=0.5$, (b) $\beta=1$
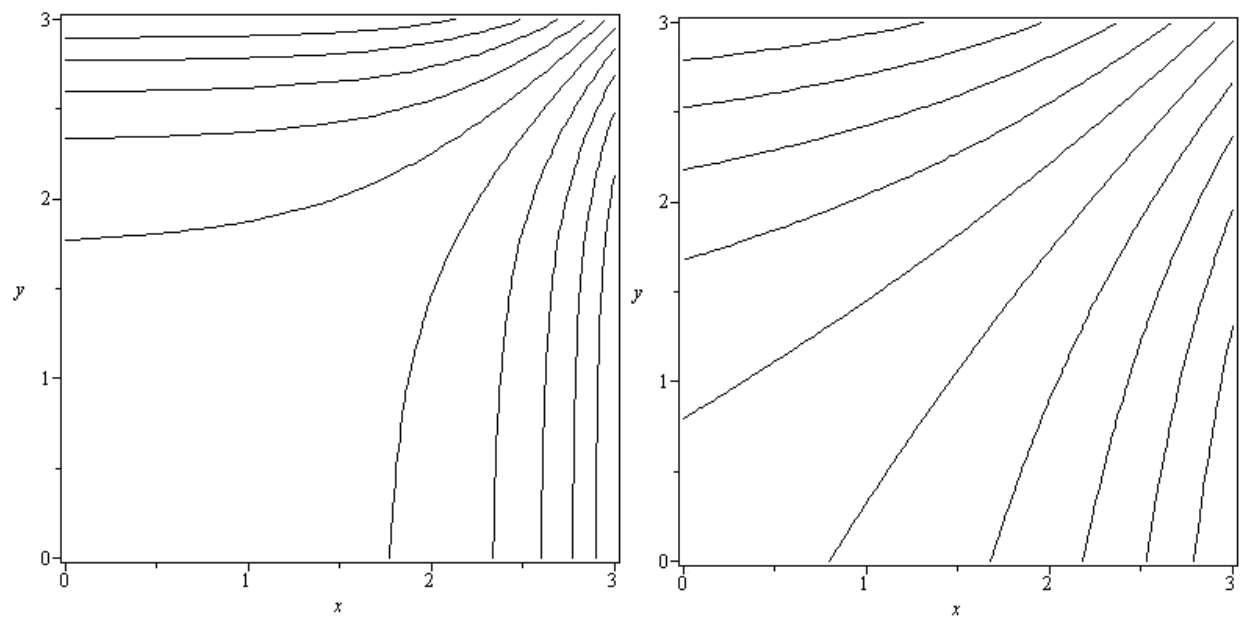

Figure 3: . Stream lines of the corner flow for (a) $\beta=0.5$, (b) $\beta=1$

behind each single element of the grid. Figure (1a) is plotted for $\beta=0.5$ and it can be observed that for smaller value of $\beta$ the size of eddies become larger. Figure (1b) which represents the viscous fluid case is plotted against $\beta=1$.

In figures $2(\mathrm{a})$ and $2(\mathrm{~b})$, the streamlines for reverse flow above a porous 

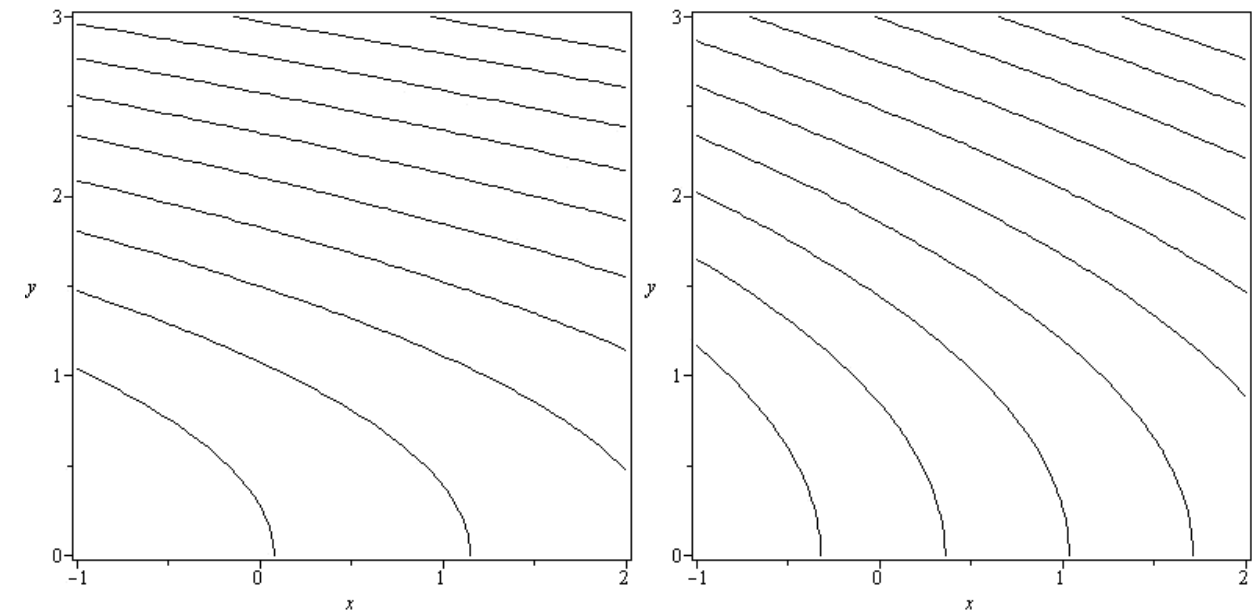

Figure 4: Stream lines for asymptotic suction flow for (a) $\beta=0.5$, (b) $\beta=1$

plate is shown. For these two graphs the values of $\beta$ are assumed respectively $\beta=0.5$ and 1 . It can be seen from figure $2(\mathrm{a})$ that for smaller value of $\beta$ the flow reversal is minimum and the effect of suction moves closer to the leading edge of the plate. Figure 2(b) indicates the viscous fluid case which also shows flow reversal.

Figures 3(a) and 3(b) represent the streamlines pattern for stretching sheet with $\beta=0.5$ and $\beta=1$. It can be seen that the stream lines formed by the stretching of plate is confined to a region, beyond which a uniform flow can be observed. Figure 3(b) shows the viscous fluid case and is plotted for $\beta=1$ It is also noted that for smaller value of $\beta$ the streamlines moves closer to the centerline of the plate.

The stream lines for the corner flow with both the walls permeable are shown in figures $4(\mathrm{a})$ and $4(\mathrm{~b})$. It is observed that as $\beta$ becomes smaller the streamlines become closer to the central part. The second figure shows the viscous fluid case for $\beta=1$.

Streamlines in figures 5(a) and 5(b) are plotted for asymptotic suction profile. 


\section{Conclusions}

Some exact solutions have been investigated for two dimensional equations of motion of the incompressible couple stress fluid by assuming vorticity as a function of stream. This study shows that each solution is strongly dependent on parameter. Solutions for the stream functions and velocity distributions studied in this work demonstrate reasonable agreement with the already existing solutions for viscous fluid found in literature [2].

\section{References}

[1] O.P. Chandna, E.O. Oku-Upkong, Flows for chosen vorticity functions: Exact solutions of the Navier-Stokes equations. Developments in Theoretical and Applied Mechanics. 17, 69(1982).

[2] P. G. Drazin and N. Riley, The Navier-Stokes Equations: a classification of flows and exact solutions. London Mathematical Society Lecture Notes. 334.

[3] M. H. Hamdan, Single-phase flow through porous channels. A review: Flow models and entry conditions, Applied Mathematics and Computation, 62, 203-222, (1994).

[4] M. H. Hamdan and F. M. Allan, A note on generalized Beltrami flow through porous media, International Journal of Applied and Pure Mathematics, 27(4), 491-499, (2006).

[5] L. I. G. Kovasznay, Laminar flow behind a two dimensional grid, Proc. Cambridge. Phil. Soc., 58-62, (1948).

[6] S. P. Lin and M. Tobak, Reversed flow above a plate with suction, AIAAJ., 24, 334-335, (1986).

[7] K. Vafai and S. J. Kim, Forced conviction in a channel filled with a porous medium: An exact solution, Journal of Heat Transfer, 111, 1103-1106, (1989). 
